SMART AGENT AND IT'S EFFECT ON ARTIFICIAL INTELLIGENCE : A REVIEW STUDY

\author{
${ }^{1}$ Renas Rajab Asaad, ${ }^{2}$ Veman Ashqi Saeed, ${ }^{3}$ Revink Masud Abdulhakim \\ ${ }^{1,3}$ Department of Computer Science, Nawroz University, Duhok, KRG - Iraq \\ ${ }^{2}$ Department of Information Technology Management, Duhok Polytechnic University, Duhok, KRG - Iraq
}

\begin{abstract}
Current networking technologies, as well as the ready availability of large quantities of data and knowledge on the Internet-based Infosphere, offer tremendous opportunities for providing more abundant and reliable information to decision makers and decision support systems. The use of the Internet has increased at a breakneck pace. Some prevailing features of the Infosphere, however, have hindered successful use of the Internet by humans or decision support machine systems. To begin with, the information available on the internet is disorganized, multi-modal, and spread around the globe on server pages. Second, every day, the number and variety of data sources and services grows dramatically. In addition, the availability, type, and dependability of information services are all changing all the time. Third, the same piece of knowledge can be obtained from a number of different sources. Fourth, due to the complex existence of information sources and possible information updating and maintenance issues, information is vague and probably incorrect. As a result, collecting, filtering, evaluating, and using information in problem solving is becoming increasingly difficult for a human or computer device. As a consequence, identifying information sources, accessing, filtering, and incorporating data in support of decision-making, as well as managing information retrieval and problem-solving efforts of information sources and decision-making processes, has become a critical challenge. To fix this issue, the idea of "Intelligent Software Agents" has been suggested. Although a precise definition of an intelligent agent is still a work in progress, the current working definition is that Intelligent Software Agents are programs that act on behalf of their human users to perform laborious information gathering tasks such as locating and accessing information from various on-line information sources, resolving inconsistencies in the retrieved information, filtering out irrelevant data.
\end{abstract}

Keywords: Artificial Intelligence, Swarm Intelligence, Smart Agent.

\title{
1. INTRODUCTION TO ARTIFICIAL INTELLIGENCE
}

Artificial intelligence (AI) is a subfield of computer science and information science. It focuses on creating hardware and software systems that solve problems and complete tasks that would be considered intelligent if completed by humans. The research and development of machines such as robots, automatic pilots for airplanes and space ships, and "smart" military arms are all part of the AI sector. Europeans prefer the word machine intelligence (MI) to artificial intelligence (AI).

Artificial intelligence theory and practice are contributing to the development of a broad variety of artificially intelligent tools. These tools can solve or assist in the solving of a growing number of problems, often under the supervision of a human and sometimes without it. AI has provided a variety of outcomes that are significant to students, teachers, our overall educational system, and our community over the last 50 years.

This short book offers a summary of AI from the viewpoints of K-12 education and teacher education. It is tailored to preservice and inservice students, as well as school administrators. This booklet, on the other hand, would be useful to educational assistants, parents, school site council members, school board members, and those who are involved in education.

This book is intended for self-study, seminars, a short course, or as a unit of study in a longer course on ICT in education. It includes a variety of ideas for bringing the content into practice right away, as well as a number of activities for use in workshops and courses. In the appendix, educators and students can find ideas for ProjectBased Learning activities.

\section{ARTIFICIAL INTELLIGENCE}

In the field of AI, there is a large amount of published research and popular literature (Artificial Intelligence-a \& b, n.d.; Minsky 1960; AI Journals \& Associations, n.d.). The term "artificial intelligence" was coined by John McCarthy for a Dartmouth University conference in 1956. (Buchanan, n.d.). 
Here are three different ways to define AI. The first comes from a pioneer in the area, Marvin Minsky. The second is by Allen Newell, a Marvin Minsky contemporary. The third description is a more recent one from 1990 , and it is very similar to the previous ones.

"Artificial intelligence is the science of making machines do things that would require intelligence if performed by men," Marvin Minsky said in the early 1960s. Minsky's contributions to Feigenbaum and Feldman (1963) include "Steps Toward Artificial Intelligence" (pp 406-450) and "A Selected Descriptor: Indexed Bibliography to the Literature on Artificial Intelligence" (pp 406-450). (pp 453-475)

Allen Newell describes intelligence as "the degree to which a system approximates a knowledge-level system" in his book Unified Theories of Cognition. The ability to bring all of a system's information to bear in the solution of a problem is known as perfect intelligence (which is synonymous with goal achievement). This is distinct from ignorance, which is described as a lack of knowledge about a specific problem space.

Artificial intelligence is essentially the application of artificial or non-naturally occurring structures that use the knowledge-level to accomplish objectives, according to this concept of intelligence. (This is where theories and hypotheses come in.)

What is artificial intelligence and how does it work? It's not always easy to come up with a description of a discipline that will please all of its practitioners. Artificial intelligence research covers a broad variety of subjects. AI, in its broadest sense, is the computer-assisted discovery of strategies for resolving difficult problems that have historically been solved by humans. Complex logical inference, diagnosis, visual recognition, natural language comprehension, game play, interpretation, and preparation are examples of such activities (Horvitz, 1990).

\section{HUMAN INTELLIGENCE}

Intelligence research and measurement have a long history. In the early 1900s, Alfred Benet and Theodore Simon, for example, created the first Intelligence Quotient (IQ) test. You've probably taken a few IQ tests and can recall a number that represented your score on one of them. Most likely, you find it odd that a single number can be used to assess a person's cognitive abilities. Many people believe that people have multiple intelligences and that no single number can adequately reflect their intellect.

IQ is a difficult term to grasp. There is no consensus among IQ experts on what constitutes intelligence or how to calculate it. (Most people are unsatisfied with the argument "IQ is what an IQ test measures.")

Howard Gardner (1993), David Perkins (1995), and Robert Sternberg (1988) are intelligence scholars who have published best-selling books. Howard Gardner is perhaps the most well-known of the three among K-12 educators. With such educators, his theory of Multiple Intelligences has proven to be very influential (Mckenzie, n.d.). Many scholars, on the other hand, have contributed to the vast and ever-growing array of intelligence research papers (Yekovich 1994).

\section{INTRODUCTION TO SMART AGENT}

A software agent that uses Artificial Intelligence (AI) to achieve its clients' objectives. Artificial intelligence is the mechanical replication of human intelligence. Clients can minimize human workload by delegating tasks to ISAs that would usually necessitate human-like intelligence.

Many researchers who used to refer to their work as "AI" are now focusing on "agent technology." As a consequence, in today's research culture, the term "agent" alone usually connotes ISAs.

For ISAs, delegation is even more absolute. ISAs have the potential to create and apply novel rules of conduct that humans do never have the time or desire to examine. Since ISAs can perform comprehensive logical planning and inference, the client-agent relationship of trust is or must be much greater, particularly when client resources are consumed for reasons unknown or several complex operations are executed before human observers can respond.

Higher-order functionality is added to the mix of capabilities by ISAs' competency. ISAs may also analyze data to identify non-obvious or secret patterns, extracting knowledge from raw data, in addition to interacting with their environment to gather data and actuate changes. Human media such as natural language text, voice, and vision are incorporated into environmental modes of interaction, making them richer.

Self-monitoring of progress against client goals, as well as continuous, online learning to enhance performance, are examples of adaptability in ISAs. Since ISAs have adaptive mechanisms, they are less vulnerable to 
environmental changes and may even improve. Furthermore, client responsiveness may go so far as to assume what a client needs when the client does not know or is unable to articulate the desired goals in concrete terms.

\section{AGENT VARIANTS}

\subsection{Mobile Agents:}

Also known as traveling agents, these programs will shuttle their being, code and state, among resources. This often improves performance by moving the agents to where the data reside instead of moving the data to where the agents reside. The alternative typical operation involves a client-server model. In this case, the agent, in the role of the client, requests that the server transmit volumes of data back to the agent to be analyzed. Oftentimes the data must be returned by the agent to the server in a processed form. Significant bandwidth performance improvements can be achieved by running the agents within the same chassis as the data. Mobile agent frameworks are currently rare, however, due to the high level of trust required to accept a foreign agent onto one's data server. With advances in technologies for accountability and immunity, mobile agent systems are expected to become more popular.

\subsection{Distributed Agents:}

Load-balancing can be achieved by distributing agents over a finite number of computational resources. Some mobile agents are self-distributing, seeking and moving to agent platforms that can offer the higher computational resources at lower costs.

\subsection{Multiple Agents:}

Some tasks can be broken into sub-tasks to be performed independently by specialized agents. Such agents are unaware of the existence of the others but nonetheless rely upon the successful operations of all.

\subsection{Collaborative Agents:}

Collaborative agents interact with each other to share information or barter for specialized services to effect a deliberate synergism. While each agent may uniquely speak the protocol of a particular operating environment, they generally share a common interface language which enables them to request specialized services from their brethren as required.

\subsection{Social Agents:}

Anthropomorphism is seen by some researchers as a key requirement to successful collaboration between humans and agents. To this end, agents are being developed which can both present themselves as human-like creations as well as interpret human-generated communications such as continuous speech, gestures, and facial expressions.

\section{INTELLIGENT SOFTWARE AGENT ARCHITECTURE}

The central functionality of such an agent-based distributed database system may be described in terms of mediator agents. They are supported by a set of wrapper agents that in addition collaborate with user interface agents, matchmaker agents, and ontology agents. The main purpose of such an architecture is to enable intelligent interoperability among heterogeneous sources and avoid centralized control of the system. So-called wrapper agents provide access to local information sources, extract content from that source and perform appropriate data conversion. In addition, a mediator may collaborate with other information agents such as broker or matchmaker agents, ontology agents, and user interface agents. In general, the mediator:

- Translates between local ontology’s (variable names, value labels, and classifications),

- Decomposes and executes complex queries on distributed relevant sources with the help of a matchmaker agent,

- $\quad$ Composes the partial responses obtained from multiple information sources, and

- $\quad$ Returns the query result to the user.

The main mediator agent in MISSION is the covering agent, which decides how the query is best covered by the candidate datasets, and the planning agent, which converts the optimal cover into a plan (defined in terms of statistical operators) and manages the execution of that plan. If only a partial match is made the matching agent may use a negotiation agent to determine if a full integration is possible. The negotiation agent is utilized to determine if different classification schemes can be mapped onto each other via a common ontology. This task is 
typically carried out using classification servers. The corresponding wrapper agents comprise the brokering agents in liaison with the costing agents and information agents. Here brokering agents have the capability of learning other retrieval strategies if there is a problem with the optimal strategy (as constructed by the covering agent). Costing agents (including authentication) are responsible for determining costs for retrieving various data fragments from possible data sources; possible costs are monetary, Internet transportation time and processing time. Information agents act on behalf of the data sources.

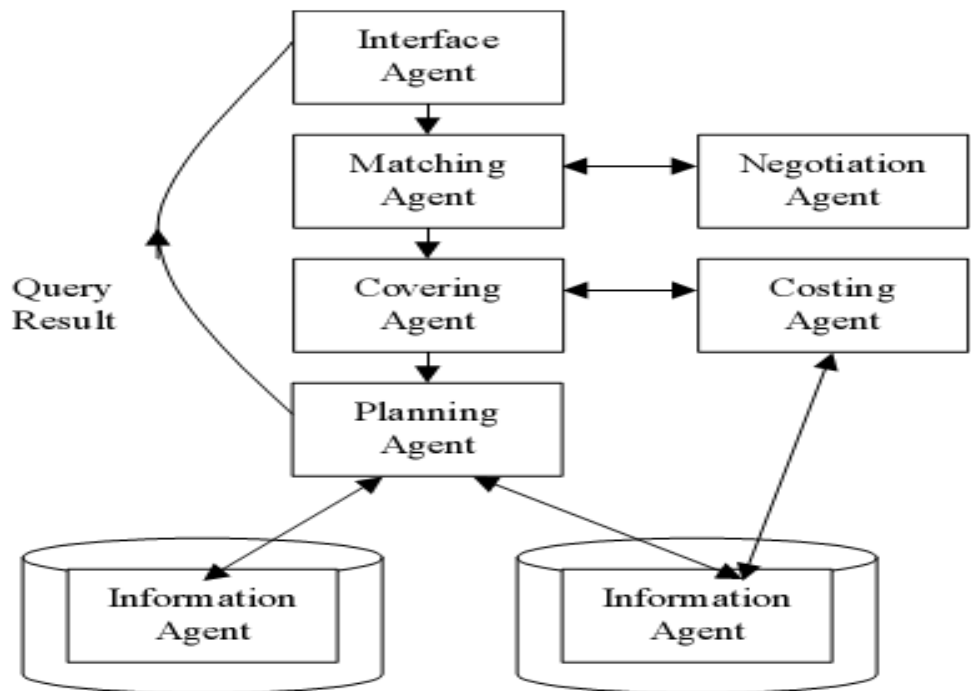

\section{CLASSIFICATION OF INTELLIGENT SOFTWARE AGENTS}

Agents can be classified according to the specific properties, or attributes, they exhibit. These include the following:

\subsection{Mobility}

This refers to the extent to which an agent can move around a network. This leads to a distinction between static and mobile agents. Sometimes this includes cloning to distribute sub-tasks in a remote environment.

\subsection{Deliberative behaviors.}

Deliberative agents possess an internal reasoning model and exhibit planning and negotiation skills when engaged with other agents in order to achieve their goals. In contrast with deliberative agents, reactive agents lack an internal reasoning model, but rather act upon the environment using a stimulus-response type of behaviors.

\subsection{Primary attributes.}

The most important attributes of an agent are referred to as primary attributes; less important, or secondary attributes, are listed below. The primary attributes include the following three:

\subsection{Autonomy:}

Reflects the ability of agents to operate on their own, without immediate human guidance, although the latter is sometimes invaluable.

\subsection{Co-operation}

Refers to the ability to exchange high-level information with other agents: an attribute which is inherent in multiple agent systems (MAS).

\subsection{Learning:}

Refers to the ability of agents to increase performance over time when interacting with the environment in which they are embedded. In agents combining several of the primary attributes are referred to by different names again autonomous agents that co-operate are called collaborative agents, those that learn are referred to as interface agents, and those that do both are termed smart agents. 


\subsection{Secondary attributes}

Agents can be classified according to a number of other attributes, which could be regarded as being secondary to the ones described above. Rather than a comprehensive list, some examples of secondary attributes that agents may exhibit will be given. Agents may be classified, for example, by their pro-active versatility - the degree to which they pursue a single goal or engage in a variety of tasks. Furthermore, one might attribute social abilities to agents, such as truthfulness, benevolence and emotions (anger, fear), although the last is certainly controversial. One may also consider mental attitudes of agents, such as beliefs, desires, and intentions (in short: BDI's).

By combining these properties and attributes, (Caglayan, A.K. and Harrison, C.G., 1997) hybrid agents and heterogeneous agents can be constructed. With hybrid agents two or more properties and/or attributes are combined in the design of a single agent. This results in the combination of the strengths of different agentdesign philosophies in a single agent, while at the same time avoiding their individual weaknesses. It is not possible to separate such an agent into two other agents. Heterogeneous agents combine two or more different categories of agents in such way that they interact via a particular communication language.

\section{THE AGENT MODEL}

With this insight into the parent disciplines of agent design, a detailed model of the internal workings of an agent can be given. First a layered model of an agent that is both deliberative, reactive and co-operating will be given. Such an agent consists of three layers. These layers are: the behaviors-based layer, the local planning layer and the co-operative planning layer. The agent also uses three models that represent different parts of reality: the world model, the mental model and the social model. The world model contains a description of the agent's environment and is linked to the behaviors-based layer. The mental model describes the inner state of the agent itself and is linked to the local planning layer. The social model describes the inner states of other agents in the environment and is linked to the co-operative planning layer.

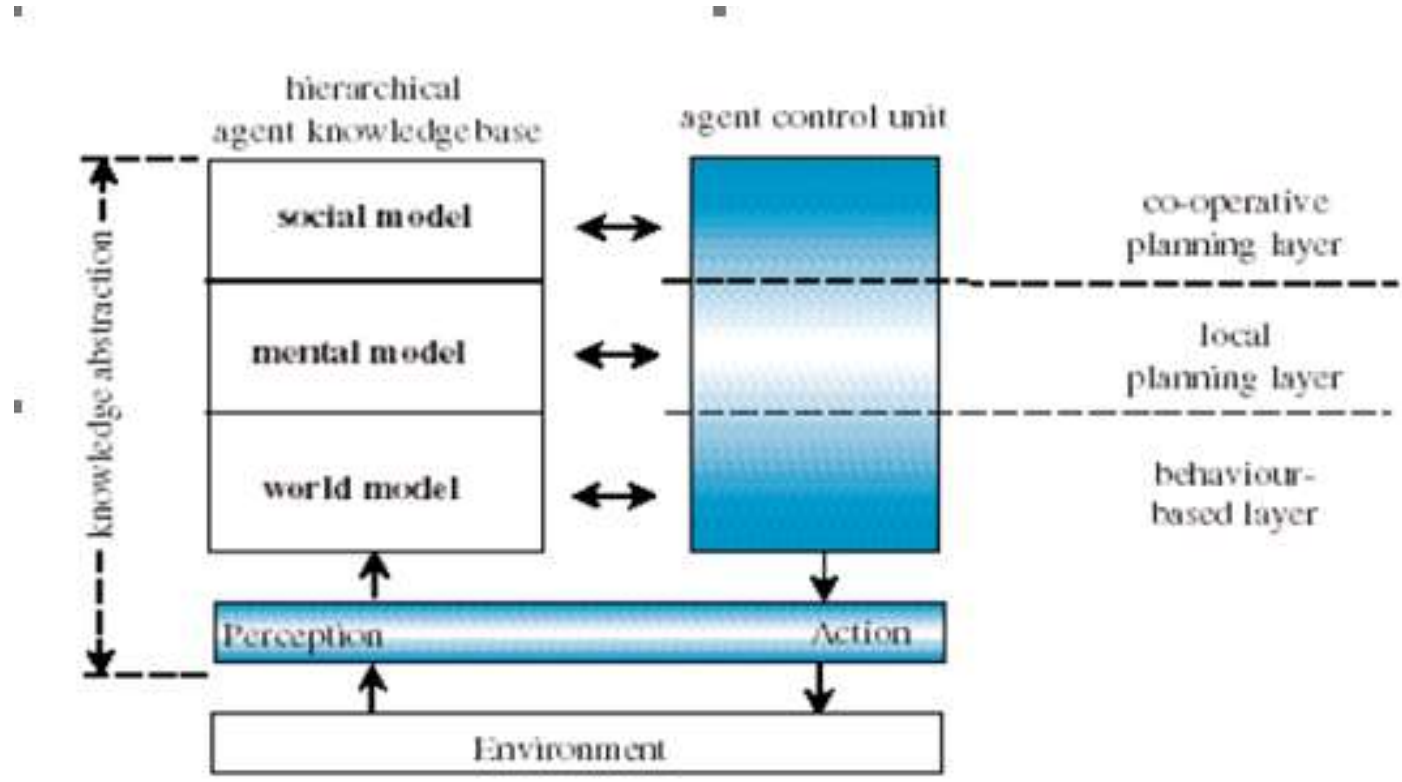

Figure 2: The layered design of an agent that is both deliberative, reactive, and co-operating

The models are hierarchical, see figure 1. The agent will use the models to interpret the input from the environment and to plan possible internal and external actions. The world model helps the agent to make decisions about its environment. The mental model helps the agent to plan possible actions the agent can perform to fulfil its task. The social model helps the agent to control actions taken by other agents in order to co-operate with these other agents and to avoid conflicts with them.

Another way to describe an agent that combines deliberative, reactive, and co-operating properties and attributes, is given in figure 2 . 


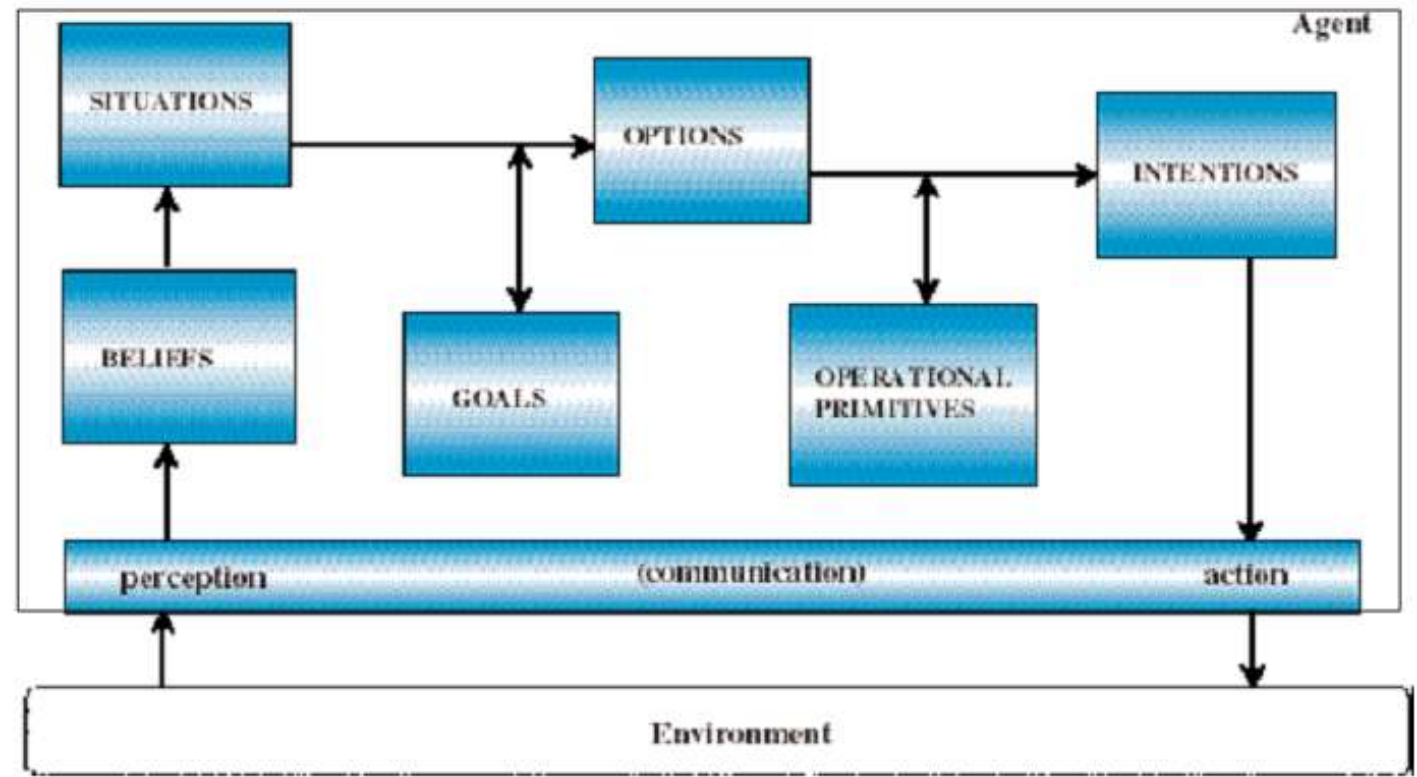

Figure 3: The agent model - a conceptual model of an agent that combines deliberative, reactive, and cooperating properties and attributes

Figure 2 depicts the agent control unit (see figure 2.3) of the hybrid agent, its conceptual building blocks, and their relationships. Conceptually, one can distinguish the following components:

1 Perception. This refers to the symbolic representation of the information communicated to the agent.

2 Beliefs. These express the expectations an agent has about the current state of the world and about the likelihood that a specific action produces certain effects.

3 Situations. These enable the agent to identify the need for activity. According to the three models there are three classes of situation. First there are the behavioural situations, which are a subset of the agent's world model. Second, there are the situations describing local planning. These situations are based both on the world model and on the mental model. Last, there are the situations that describe co-operative planning. These situations are based on the social model.

4 Goals. It is possible that an agent has a set of goals. These goals are context-independent. Goals can be classified into reaction goals, local goals and co-operative goals. Reaction goals are goals that are triggered by external events. These goals require a fast reaction and are of short-term base. Local goals refer to the goals of the agent itself. Co-operative goals are goals that are shared among a group of different agents.

5 Options. An agent can also contain a set of options. The options represent the agent's motivational state. Based on the current situation, a set of context-dependent options is selected. These options are related to the agent's goals. Given the selected option, operational primitives are selected to achieve the current goal(s).

6 Operational primitives. These primitives or software techniques enable an agent to achieve certain goals. Once selected, these operational primitives are merged into an execution schedule.

7 Intentions. An agent also has intentions. These intentions define the action an agent is going to take (the deliberative state of the agent). The intentions lead to the execution of the operational primitives from the execution schedule.

\section{THE COMMUNICATION MODEL}

To make communication possible, all agents need to be active in the same network infrastructure. This network architecture needs to contain one or more of the following facilities:

- $\quad$ facilities to run an agent (program);

- $\quad$ facilities to support communication between agents of different types;

- $\quad$ facilities to allow movement of agents from one system to another; 
facilities to allow cloning of a mobile agent in a local environment;

facilities to encapsulate agent information;

facilities to identify and authenticate other agents.

There is no need for agents to stay in the same place. If it is more efficient to accomplish a task or achieve an objective at a different location the agent might move to that location. Following presents a black box model of

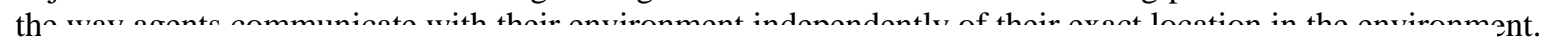

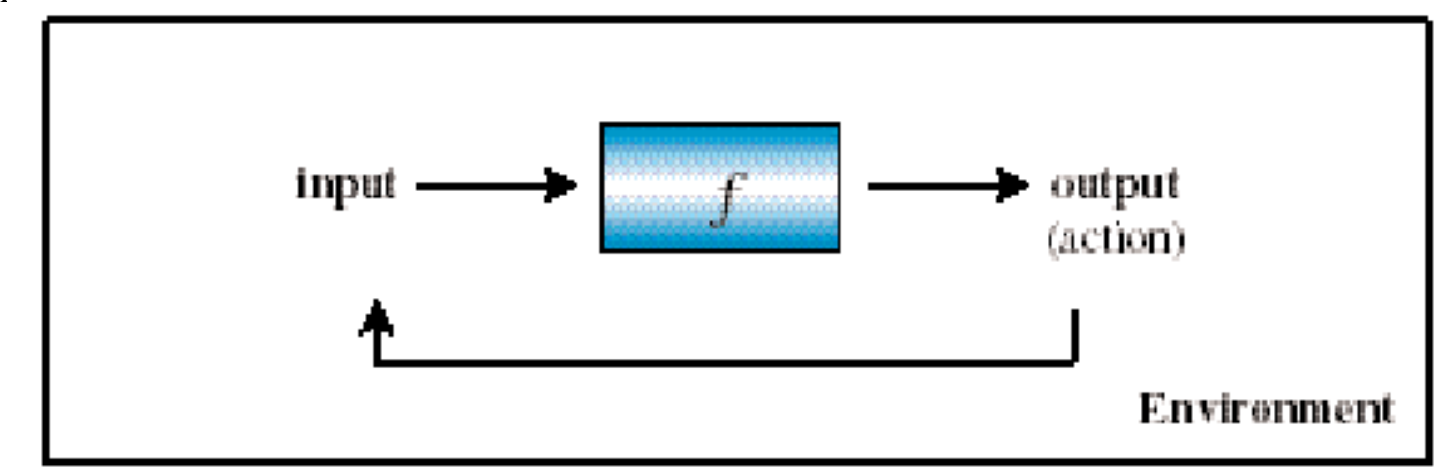

\section{APPLICATIONS}

To date, ISAs have been successfully employed in multiple application endeavors, some of which are listed below.

- Data Collection and Filtering

- Pattern Recognition

- $\quad$ Event Notification

- Data Presentation

- $\quad$ Planning and Optimization

- $\quad$ Rapid Response Implementation

11. FUTURE AREAS OF APPLICATION

1. Systems and Network Management

2. Mobile Access / Management

3. Mail and Messaging

4. Information Access and Management

5. Collaboration

6. Workflow and Administrative Management

7. Electronic Commerce

8. $\quad$ Adaptive User Interfaces

\section{SWARM INTELLIGENCE}

Swarm intelligence (SI) is in the field of artificial intelligence (AI) and is based on the collective behavior of elements in decentralized and self-organized systems. SI has a great involvement in the field of Internet of Things (IoT) and IoT-based systems in order to logically control their operations. As IoT-based systems are complex and consist of smart objects, so powerful decentralized algorithms supported by SI are used to resolve such complex problems. Intelligent algorithms of SI can solve the challenging issues of IoT systems due to their dynamic properties, device mobility, wireless communication, and information provision. SI algorithms are well established and applied in well-known aptitude-based problems where real-time actions are handled efficiently. SI algorithms like ant colony optimization, artificial bee colony, and social spider optimization play important 
roles in normalizing the IoT processes. The current chapter covers the technical understanding of the execution of SI and performs an analytical review of the technology-based advanced applications of IoT supported by very logical SI approaches.

\section{CONCLUSION}

"The notion of Intelligent Software Agents (ISA) capable of finding, organizing, and analyzing information on behalf of their owners is the most important concept regarding the Internet today. This new generation of agent technology promises to help reduce the cost, effort, and time of filtering information on the Web."

In the near future, we will be making some very significant choices in our relation to and use of technology for learning and development. New tools for data visualization and intelligent agents aren't too unsettling; at best, the issue becomes one of personal privacy and who we give access to our information habits. The bigger issue relates to technology augmentation - implants, real-time tracking, smart pharmaceuticals, bio-technical convergence, and nanotechnology will force a reconsideration of what it means to be human. Oh, what fun we shall have with the ethical considerations.

\section{REFERENCES}

[1] Russell, S., \& Norvig, P. (2002). Artificial intelligence: a modern approach.

[2] Asaad, R. R., Ahmad, H. B., \& Ali, R. I. (2020). A Review: Big Data Technologies with Hadoop Distributed Filesystem and Implementing M/R. Academic Journal of Nawroz University, 9(1), 25-33.

[3] Mitchell, R. S., Michalski, J. G., \& Carbonell, T. M. (2013). An artificial intelligence approach. Berlin: Springer.

[4] Marashdih, A. W., Zaaba, Z. F., \& Almufti, S. M. (2018). The Problems and Challenges of Infeasible Paths in Static Analysis. International Journal of Engineering \& Technology, 7(4.19), 412-417.

[5] Almufti, S. M., \& Shaban, A. A. (2018). U-Turning Ant Colony Algorithm for Solving Symmetric Traveling Salesman Problem. Academic Journal of Nawroz University, 7(4), 45-49.

[6] Asaad, R. R. (2014). An Investigation of the Neuronal Dynamics Under Noisy Rate Functions (Doctoral dissertation, Eastern Mediterranean University (EMU)-Doğu Akdeniz Üniversitesi (DAÜ)).

[7] Almufti, S., Asaad, R., \& Salim, B. (2018). Review on Elephant Herding Optimization Algorithm Performance in Solving Optimization Problems. International Journal of Engineering \& Technology, 7, 6109-6114.

[8] Karaboga, D., \& Akay, B. (2009). A survey: algorithms simulating bee swarm intelligence. Artificial intelligence review, 31(1-4), 61.

[9] Asaad, R. R., \& Abdulnabi, N. L. (2018). Using Local Searches Algorithms with Ant Colony Optimization for the Solution of TSP Problems. Academic Journal of Nawroz University, 7(3), 1-6.

[10] Almufti, S., Marqas, R., \& Asaad, R. (2019). Comparative study between elephant herding optimization (EHO) and U-turning ant colony optimization (U-TACO) in solving symmetric traveling salesman problem (STSP). Journal Of Advanced Computer Science \& Technology, 8(2), 32.

[11] Asaad, R. R., \& Ali, R. I. (2019). Back Propagation Neural Network (BPNN) and sigmoid activation function in multi-layer networks. Academic Journal of Nawroz University, 8(4), 216-221.

[12] Yuen, H. C. (2006). U.S. Patent No. 7,003,792. Washington, DC: U.S. Patent and Trademark Office.

[13] Asaad, R. R. (2019). Güler and Linaro et al Model in an Investigation of the Neuronal Dynamics using noise Comparative Study. Academic Journal of Nawroz University, 8(3), 10-16.

[14] Kennedy, J. (2006). Swarm intelligence. In Handbook of nature-inspired and innovative computing (pp. 187-219). Springer, Boston, MA.

[15] Almufti, S., Marqas, R., \& Ashqi, V. (2019). Taxonomy of bio-inspired optimization algorithms. Journal Of Advanced Computer Science \& Technology, 8(2), 23.

[16] Abdulrahman, S. M. (2017). Using Swarm Intelligence for solving NP-Hard Problems. Academic Journal of Nawroz University, 6(3), 46-50. 
[17] Sutton, J. (1997). One smart agent. The Rand Journal of Economics, 605-628.

[18] Asaad, R. R., Sulaiman, Z. A., \& Abdulmajeed, S. S. (2019). Proposed System for Education Augmented Reality Self English Learning. Academic Journal of Nawroz University, 8(3), 27-32.

[19] Marqas, R. B., Almufti, S. M., Othman, P. S., \& Abdulrahma, C. M. Evaluation of EHO, U-TACO and TS Metaheuristics algorithms in Solving TSP.

[20] Zebari, A. Y., Almufti, S. M., \& Abdulrahman, C. M. Bat algorithm (BA): review, applications and modifications.

[21] Asaad, R. R., \& Abdulhakim, R. M. (2021). The Concept of Data Mining and Knowledge Extraction Techniques. Qubahan Academic Journal, 1(2), 17-20.

[22] Nilsson, N. J. (2014). Principles of artificial intelligence. Morgan Kaufmann.

[23] Cli, D. (1997). Minimal-intelligence agents for bargaining behaviors in market-based environments. Hewlett-Packard Labs Technical Reports.

[24] Poole, D. L., \& Mackworth, A. K. (2010). Artificial Intelligence: foundations of computational agents. Cambridge University Press.

[25] Russell, S., \& Norvig, P. (2002). Artificial intelligence: a modern approach.

[26] Huhns, M. N., \& Singh, M. P. (Eds.). (1998). Readings in agents. Morgan Kaufmann.

[27] Pavón, J., \& Corchado, J. (2004). Agents for the web. International journal of Web engineering and technology, 1(4), 393-396.

[28] Asaad, R. R. (2021). Review on Deep Learning and Neural Network Implementation for Emotions Recognition. Qubahan Academic Journal, 1(1), 1-4.

[29] Othman, P. S., Ihsan, R. R., Marqas, R. B., \& Almufti, S. M. (2020). Image Processing Techniques for Identifying Impostor Documents Through Digital Forensic Examination. Image Processing Techniques, 62(04). 